BMJ Surgery, Interventions, $\&$ Health Technologies

\section{The Precision Prostatectomy: an IDEAL Stage 0, 1 and 2a Study}

To cite: Sood A, Jeong W, Taneja K, et al. The Precision Prostatectomy: an IDEAL Stage 0, 1 and 2a Study. BMJ Surg Interv Health Technologies 2019;1:e000002. doi:10.1136/ bmjsit-2019-000002

Received 18 March 2019 Revised 17 July 2019 Accepted 17 July 2019

\section{Linked}

- http://dx.doi.org/10.1136/ bmjsit-2019-000015

Check for updates

(c) Author(s) (or their employer(s)) 2019. Re-use permitted under CC BY-NC. No commercial re-use. See rights and permissions. Published by BMJ.

${ }^{1}$ Vattikuti Urology Institute, Henry Ford Hospital, Detroit, Michigan, USA

${ }^{2}$ Department of Pathology, Henry Ford Hospital, Detroit, Michigan, USA

Correspondence to

Dr Akshay Sood;

asood1@hfhs.org

\begin{abstract}
Objective This study aimed to develop a preclinical model of prostate cancer (CaP) for studying focal/hemiablation of the prostate (IDEAL stage 0 ), and to use the information from the stage 0 investigation to design a novel focal surgical treatment approach-the precision prostatectomy (IDEAL stage 1/2a).

Methods The IDEAL stage 0 study included simulation of focal/hemiablation in whole-mount prostate specimens obtained from 100 men who had undergone radical prostatectomies, but met the criteria for focal/ hemiablation. The IDEAL stage 1/2a was a prospective, single-arm, Institutional Review Board-approved study of precision prostatectomy undertaken in eight men, who met the predetermined criteria. Criteria for both stages included (1) prostate-specific antigen (PSA) $\leq 15 \mathrm{ng} /$ $\mathrm{mL}$, (2) stage $\leq \mathrm{cT} 2$, (3) dominant unilateral lesion with Gleason $\leq 4+3$ with any number of cores or $\%$ cores involved ipsilaterally on transrectal biopsy, (4) no primary Gleason $\geq 4$ contralaterally on transrectal biopsy, and (5) preoperative erectile function score (International Index of Erectile Function (IIEF)-5) of $\geq 17$ (out of 25) without PDE5i (applicable only to the stage 1/2a study participants). Feasibility and safety of the precision prostatectomy technique, and short-term urinary, sexual and oncological outcomes were studied.
\end{abstract}

Results Analysis of whole-mount specimens in the 100 men showed an index lesion (>1 cm in diameter) in all. Ninety-eight men had satellite lesions smaller than $0.5 \mathrm{~cm} \wedge 3$ in volume -46 on the side of the dominant lesions and 52 in the contralateral lobe. If the men in this modeling cohort had undergone focal ablation with a 5-10 mm untreated margin, all except one would have had at least Gleason 6 residual cancer. If they had undergone hemiablation with no untreated tissue on the ablated side, 56 men would have had residual cancer on the contralateral side, of whom 21 would have had clinically significant cancer (Gleason 7 or higher). If these men had undergone precision prostatectomy, with preservation of $5-10 \mathrm{~mm}$ of tissue on the non-dominant side, $10 \%$ and $4 \%$ would have had Gleason $3+4$ and Gleason $4+3$ disease left behind, respectively. For the stage 1/2a study, the median (IQR) age, PSA and IIEF-5 scores at the time of surgery were 54 (52-57) years, $4.4(3.8-6.1) \mathrm{ng} / \mathrm{mL}$ and 24 (23-25), respectively. All eight patients were continent and sexually active at 12 months with a median IIEF-5 score of 21 (out of 25). At 24-30 months from surgery, the median PSA was 0.2 (range $0.1-0.7$ ) $\mathrm{ng} / \mathrm{mL}$. Six men had undergone follow-up protocol biopsies, two, with undetectable PSA, had refused. Two men had residual Gleason $3+3$ cancer, with PSA of 0.7 and $0.4 \mathrm{ng} / \mathrm{mL}$, and

\section{Key messages}

What is already known about this subject?

- Whole-gland treatment of prostate cancer (CaP) often leads to unintended adverse functional effects, in particular, sexual impotence.

- Focal ablative techniques for treatment of localized $\mathrm{CaP}$ have recently emerged to avoid such functional decline; although these focal ablative techniques have shown promise, a few limitations have emerged: (1) an inability or reluctance to treat a prostate gland $>40 \mathrm{gm}$, (2) an inability to ablate $>60 \%$ of the whole gland, (3) lack of pathological information, and (4) a high positive biopsy rate in the residual prostate tissue resulting in a high rate of redo procedures ( $25 \%$ of the patients).

What are the new findings?

- Precision prostatectomy allows for removal of greater than $90 \%$ of the prostate with complete removal of the side of the dominant lesion, and removal of the majority of satellite lesions on the contralateral side.

- All eight patients who underwent precision prostatectomy in this exploratory study were continent and potent within 12 months from surgery, and no patient (out of eight) had clinically significant residual cancer or required secondary treatment at a follow-up of 30 months.

How might it impact on clinical practice in the foreseeable future?

- Precision prostatectomy may allow patients to choose a risk-stratified surgical approach to treatment of localized CaP.

remain on active surveillance. No man has undergone secondary whole-gland therapy.

Conclusions Examination of whole-mount radical prostatectomy specimens in men who fit the conventional criteria of focal/hemiablation showed that approximately $21 \%-68 \%$ of men would have clinically significant CaP in the untreated tissue. In a small development cohort, precision prostatectomy was technically feasible, with excellent postoperative functional recovery. At 30 months of follow-up, no patient had clinically significant residual cancer or required secondary treatment. Pending longterm follow-up, a risk-stratified surgical approach may avoid whole-gland therapy and preserve erectile function in the majority of men with intermediate-risk $\mathrm{CaP}$. 


\section{INTRODUCTION}

Whole-gland treatment of localized prostate cancer ( $\mathrm{CaP})$ is associated with a substantial risk of adverse outcomes, in particular of erectile dysfunction. ${ }^{12}$ In an attempt to minimize this, a few investigators have developed techniques of focal CaP ablation, following the well-established organ-preserving treatment paradigms in other malignancies. $^{3-6}$ While several techniques have been described, ${ }^{7}$ the underlying premise is to treat the index lesion, ${ }^{89}$ where visible on imaging (with focal therapy), or one side of the prostate, when lesions are not visible (hemiablation).

Clinical studies of focal therapy in CaP, notably of high-intensity focused ultrasound (HIFU), have shown promise1-year continence and potency rates have been reported to be $100 \%$ and $89 \%$, respectively, ${ }^{10}$ and 5-year metastasis-free and cancer-specific survival rates were reported to be $98 \%$ and $100 \%$, respectively, in a recent multi-institutional study of HIFU. ${ }^{12}$ However, certain limitations have emerged: an inability or reluctance to treat a prostate gland $>40 \mathrm{~g},(2)$ an inability to ablate $>60 \%$ of the whole gland, (3) lack of pathological information on the ablated prostatic tissue, and (4) a high positive biopsy rate in the residual prostate tissue resulting in a high rate of redo procedures ( $25 \%$ of the patients) at 3 years. ${ }^{13}$

We undertook a preclinical study to model the oncological outcomes of focal/hemiablation in a cohort of men who had undergone whole-gland radical prostatectomy for localized CaP, but met the criteria for focal/hemiablation (IDEAL stage 0). We used these data to develop a technique that would optimize the maintenance of erectile function, while allowing for the maximal removal of CaP, including the index and most/all satellite lesions (IDEAL stage 1/2a). Our primary intent was the precise preservation of erectogenic nerves, hence the term precision prostatectomy. These studies follow the IDEAL framework for safe surgical innovation ${ }^{14-16}$ where preclinical studies are considered stage 0 , and the first stage of innovation, performed on a handful of carefully selected patients, stage $1 / 2 \mathrm{a}$.

\section{METHODS}

IDEAL stage 0: conceptualization of the technique and preclinical simulation

We retrospectively analyzed 100 consecutive whole-mount radical prostatectomy specimens from patients that had undergone robotic radical prostatectomy (January 2016January 2017) and satisfied the following criteria: (1) prostate-specific antigen (PSA) $\leq 15 \mathrm{ng} / \mathrm{mL}$, (2) stage $\leq$ cT2, (3) dominant unilateral lesion with Gleason score $\leq 4+3$ with any number of cores or $\%$ cores involved ipsilaterally on transrectal ultrasound (TRUS) prostate biopsy, and (4) no primary Gleason score $\geq 4$ contralaterally on TRUS prostate biopsy; these inclusion criteria were based on prior focal therapy studies. ${ }^{10} 17$ In the IDEAL stage 0 part of the study, we examined the whole-mount radical prostatectomy specimens, to
1. Map the CaP lesions, including grade and tumor diameter.

2. Determine the distribution of lesions that were below the size limit of mp-MRI detection, usually defined as $10 \mathrm{~mm}$ in diameter or $0.5 \mathrm{~cm} \wedge 3 .^{18}$

3. Simulate a "what if" scenario to examine residual cancer rates, if these men were treated with focal or hemiablation rather than with conventional prostatectomy.

4. Use the risk estimates generated in this stage to develop a novel technique-the precision prostatectomythat removes the entire hemi prostate and seminal vesicle complex on the side of the index lesion, but leaves a thin rim of prostatic capsule $(5-10 \mathrm{~mm})$ on the side contralateral to the dominant lesion.

\section{IDEAL stage 1/2a: study population, informed consent and surgical technique}

We examined the feasibility of precision prostatectomy in eight carefully selected men who were desirous of focal therapy to the prostate (IDEAL stage $1 / 2 \mathrm{a}$ ). These men were evaluated and treated over a 6-month period, between January and July 2017, allowing a minimum follow-up of 24 months. All men placed high priority on preservation of erectile function and sought out the treating surgeon specifically requesting focal therapy. No man was a candidate for active surveillance, and all met the following criteria: (1) PSA $\leq 15 \mathrm{ng} / \mathrm{mL}$, (2) stage $\leq$ cT2, (3) dominant unilateral lesion with Gleason score $\leq 4+3$ with any number of cores or $\%$ cores involved ipsilaterally on TRUS prostate biopsy, (4) no primary Gleason score $\geq 4$ contralaterally on TRUS prostate biopsy, and (5) preoperatively potent without phosphodiesterase type 5 (PDE-5) inhibitors. Data collection was under an ongoing protocol approved by the Institutional Review Board, and in compliance with Health Insurance Portability and Accountability Act of 1996 (HIPAA) regulations. Informed consent was obtained from all patients. The patients were apprised of the experimental nature of the procedure, and the fact that the risks could not be accurately estimated given the novelty of the procedure. Patients were required to review the informational material for at least 7 days before they were considered for precision prostatectomy. Failing this in-home evaluation, patients were assigned to conventional radical prostatectomy.

Patient positioning, port placement, developing the space of Retzius and prostatic pedicle dissection followed our standard anterior Vattikuti Urology Institute technique of prostatectomy. ${ }^{1920}$ However, in the precision prostatectomy, standard nerve-sparing radical prostatectomy was undertaken on the side of the dominant lesion. On the contralateral side, the dissection was started anterior to the vas deferens/seminal vesicle complex, preserving all layers of Denonvilliers' fascia, with the included erectogenic nerves. ${ }^{21} 22$ The dissection was continued $5-10 \mathrm{~mm}$ into the prostatic capsule, deliberately attempting to leave behind a thin rim of prostatic capsule $(5-10 \mathrm{~mm})$ along with the seminal vesicle/ejaculatory duct complex. 
Systematic needle biopsies (via a suprapubic approach) were taken from the remnant prostatic tissue, and sent for frozen section analysis. Completion prostatectomy was performed if the frozen biopsies showed residual cancer. Vesicourethral anastomosis was performed as previously described. ${ }^{19} 20$ The patients received a Foley or suprapubic tube per patient choice.

For the stage 1/2a study, our goals were to assess:

1. The safety and feasibility of the technique.

2. Continence and potency outcomes.

3. Short-term oncological control as assessed by biochemical recurrence (using the American Urological Association (AUA) definition of biochemical recurrence following radical prostatectomy or the ASTRO criteria used for radiation or focal therapy) and protocol biopsies of residual tissue.

\section{IDEAL stage $\mathbf{0}$ and 1/2a: variables, endpoints and definitions}

For each patient (stage 1/2a), the following clinical parameters were noted: age at surgery, body mass index, comorbidities, American Society of Anesthesiologists score, preoperative PSA, clinical tumor stage (cT), and biopsy Gleason score, total number of cores on biopsy, number of positive cores on biopsy and percentage core positivity. Operative parameters collected included total operative time, console operative time, estimated blood loss, intraoperative complications and need to convert to radical prostatectomy. Pathological parameters collected included pathologic Gleason Score, pathological tumor stage (pT) and surgical margin status (negative vs positive). The prostatectomy specimens were sectioned and processed according to the previously described methodology by Ruijter et $a l .^{23}$ Briefly, after fixation and marking of surgical planes with dye, radical prostatectomy specimens were cut into serial parallel transverse $4 \mathrm{~mm}$ thick slices. Following histological processing and complete embedding, $4 \mu \mathrm{m}$ tissue sections were stained with H\&E. All the tumor nodules were outlined in ink on the coverslips of all the slides. The whole tumor was mapped and reconstructed, and the three-dimensional (3D) shape and size of the tumor was determined. For calculating the maximum dimension of tumor, apart from the largest dimension on a single slide, the longitudinal contiguous tumor dimension across sequential sections was also taken into account. This dimension was calculated by multiplying the thickness of slice by the number of sections in which tumor was present. Postoperative complications noted included need for blood transfusion, urinary tract infections, lymphoceles, deep venous thrombosis (DVT), pulmonary embolus (PE), pneumonia, myocardial infections and death for 30 days after surgery. Preoperative and postoperative urinary and sexual function assessments were performed using the International Prostate Symptom Score (IPSS) and International Index of Erectile Function (IIEF-5) questionnaires. Postoperative PSA was collected at 4, 8 and 12 months postoperatively. Biochemical recurrence (BCR) was defined using American Urological Association (AUA) criteria for
BCR following radical prostatectomy. ${ }^{24}$ All patients were followed for at least 12 months.

Definitions: for stages 0 and $1 / 2 \mathrm{a}$, unilateral disease (preoperative) was defined as cancer limited to only one side of the prostate on 12 or more cores of TRUS-guided biopsy, while bilateral disease included both sides (the same definition was utilized for whole-mount analysis). A dominant lesion on biopsy was defined as the lesion with the highest grade, and in case, two or more lesions had the same Gleason score, the lesion with the greatest core length positivity was designated as the dominant lesion. A dominant nodule was the lesion with highest grade on final pathology, and similar to the dominant lesion on biopsy, if two or more nodules had the same Gleason score, the larger nodule was designated as the dominant nodule. Clinically significant cancer was defined as a lesion with Gleason score $\geq 3+4$, based on prior focal therapy studies. ${ }^{1011}$

Nomenclature: A focus group of 30 men, including those in this report, were asked to come up with a name for the procedure or to choose from a menu of options: focal surgery, focal prostatectomy, subtotal prostatectomy, precision surgery, precision prostatectomy or capsule preserving prostatectomy. The consensus of the men was precision prostatectomy (29/30 men), with term "Menon" added on by patient request because a majority wanted to assign the credit (or the blame) to the treating surgeon (Menon precision prostatectomy (MPP)).

\section{Statistical analyses}

Descriptive statistics of categorical variables focused on frequencies and proportions. Medians and (IQR) were reported for continuously coded variables. Kaplan-Meier estimates were constructed to assess 12-month urinary continence and sexual potency rates. Statistical analyses were performed using the $\mathrm{R}$ statistical package ( $\mathrm{R}$ Foundation for Statistical Computing, Vienna, Austria), considering a statistical significance at two-sided $\mathrm{p}<0.05$.

\section{RESULTS}

\section{IDEAL stage 0: baseline characteristics}

The median (IQR) age and PSA were 61 (58-66) years and $5.1(4.1-7.8) \mathrm{ng} / \mathrm{mL}$, respectively, in the 100 patients in the preclinical study. The clinical stage was cT1 in $79 \%$ $(\mathrm{n}=79)$ of the patients, and cT2 in the remaining. The biopsy Gleason was $3+3$ in $21 \%(n=21), 3+4$ in $53 \% \quad(n=53)$ and $4+3$ in $26 \%(\mathrm{n}=26)$. The median (IQR) number of cores positive on biopsy were 4 (3-6). On transrectal biopsies, 48 men had disease located to one half of the prostate and 52 had bilateral disease.

IDEAL stage 0: whole-mount radical prostatectomy specimen analysis, and MPP and focal HIFU simulation analysis

In prostate mapping studies of the whole-mount radical prostatectomy specimens, $96 \%$ of the patients had cancers involving at least a part the peripheral zone, defined as within $5-10 \mathrm{~mm}$ of the prostatic capsule; the remaining 


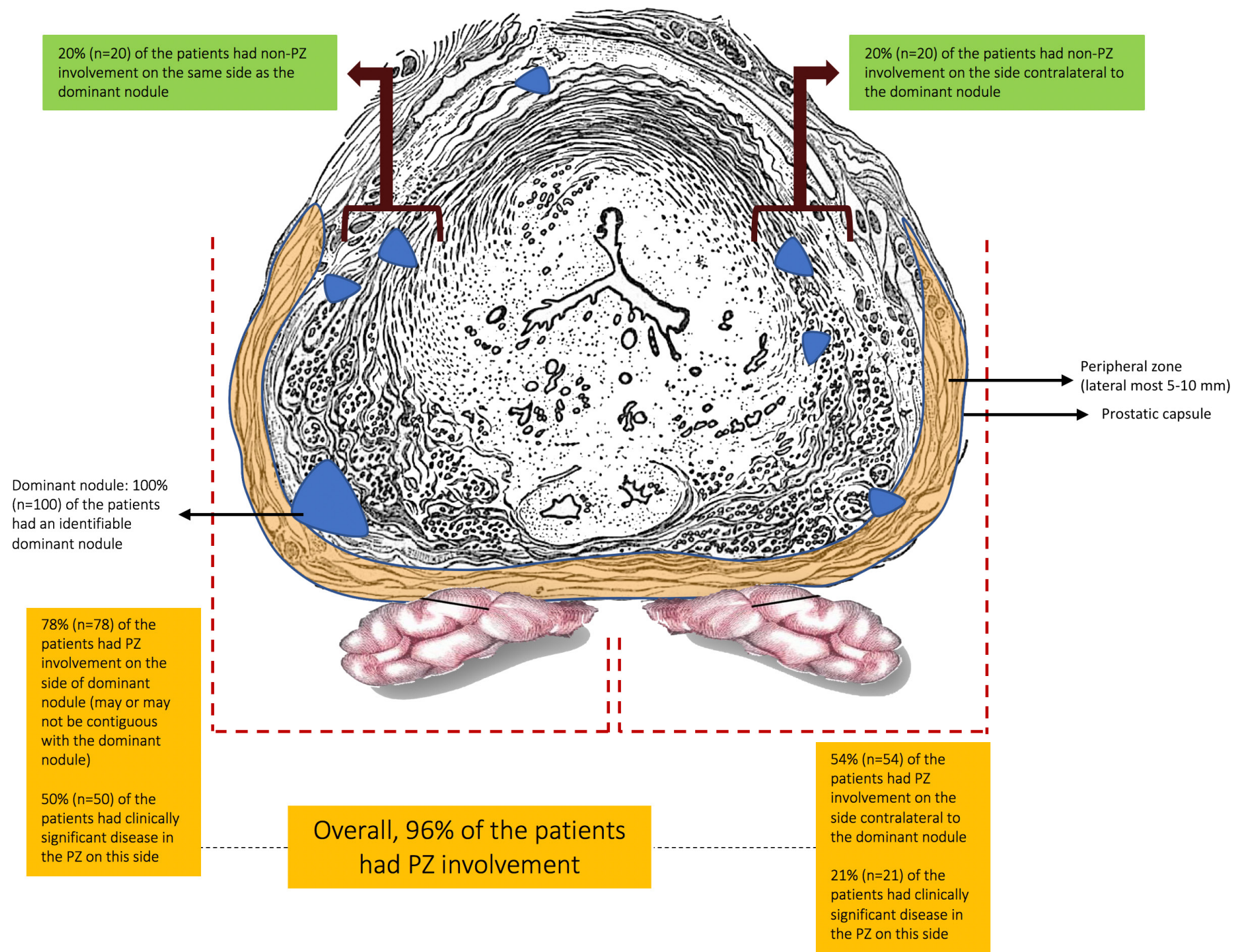

Figure 1 Whole-mount analysis and mapping of cancer lesions within the prostate in 100 radical prostatectomy specimens of patients that met criteria* for focal therapy but had undergone radical prostatectomy previously. *Inclusion criteria: (1) prostate-specific antigen $\leq 15 \mathrm{ng} / \mathrm{mL}$, (2) stage $\leq \mathrm{cT} 2$, (3) dominant unilateral lesion with Gleason score $\leq 4+3$ with any number of cores or \% cores involved ipsilaterally on transrectal ultrasound (TRUS) prostate biopsy, and (4) no primary Gleason score $\geq 4$ contralaterally on TRUS prostate biopsy; the yellow zone represents $5-10 \mathrm{~mm}$ margin of the prostatic peripheral zone and prostatic capsule.

$4 \%$ patients had anterior zone tumors only. Seventy-one per cent $(71 \%)$ of the men had clinically significant disease (Gleason score $\geq 7$ ) in the peripheral zone, within $5-10 \mathrm{~mm}$ of the prostatic capsule (figure 1).

In all 100 patients, a dominant nodule was identifiable. Ninety-eight men had additional satellite lesions smaller than $0.5 \mathrm{cc}$ in volume -46 on the side of the dominant lesions and 52 in the contralateral lobe. Seventy-two per cent $(72 \%)$ of the patients had bilateral disease on wholemount analysis (figure 1 provides further details).

If all 100 men in the modeling cohort had been treated with focal ablation, all men except 1 would have had residual Gleason $\geq 6$ cancer. Further, only 25 patients would have qualified for focal therapy, given the recommended prostate weight cut-off of $40 \mathrm{~g}$ for HIFU. In these 25 men, $44 \%$ would have had residual Gleason $3+4,16 \%$ Gleason 4+3, $4 \%$ Gleason $4+4 \%$ and $4 \%$ Gleason $4+5$ disease (figure 2), given that focal HIFU spares $5-10 \mathrm{~mm}$ of peripheral tissue on the treatment side and does not treat the contralateral side. Thus, only 8 men of the original 100 would have been eligible and completely treated in the modeling cohort. With hemiablation, 44 men would have had complete cancer ablation, 56 men would have had residual cancer and 21 would have clinically significant cancer (Gleason 7 or higher).

In the simulation analysis, if these men had undergone MPP with preservation of $5-10 \mathrm{~mm}$ of tissue on the contralateral side, no patient of the 48 with unilateral disease would have had clinically significant cancer ( $\geq$ Gleason 7 ) in the preserved tissue. If patients with bilateral disease $(n=52)$ had undergone MPP, 19.2\% would have had Gleason $3+4 \%$ and $7.6 \%$ Gleason $4+3$ disease left behind (figure 3). Hence, overall in these 100 patients, $14 \%$ of the patients would have had clinically significant (Gleason 3+4, $n=10$, Gleason $4+3, n=4$ ) disease 


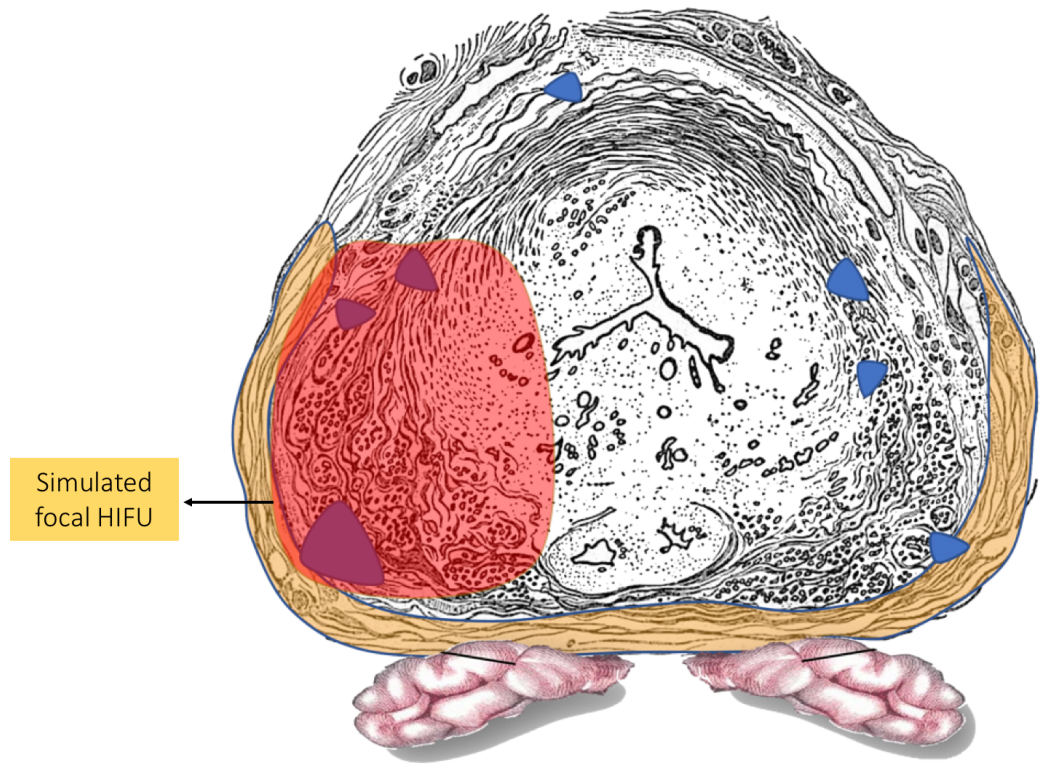

\begin{tabular}{|c|c|}
\hline Analysis of whole-mount prostatectomy with focal therapy* & $n=25$ \\
\hline Patient with no cancer left behind & 1 \\
\hline Patient with cancer left behind & 24 \\
\hline \multicolumn{2}{|l|}{ Clinically non-significant } \\
\hline $3+3$ & $7(0,7)$ \\
\hline \multicolumn{2}{|l|}{ Clinically significant } \\
\hline $3+4$ & $11(8,3)$ \\
\hline $4+3$ & $4(4,0)$ \\
\hline $4+4$ & $1(1,0)$ \\
\hline $4+5$ & $1(1,0)$ \\
\hline
\end{tabular}

\footnotetext{
*Patients fulfilling the criteria of $40 \mathrm{~g}$ prostate weight for focal HIFU; only the dominant nodule was treated in this simulation -- where the dominant nodule was within $5 \mathrm{~mm}$ of the edge of prostate capsule, a part of the dominant nodule was considered not treated in this simulation

- 14 patients had dominant nodules within $5 \mathrm{~mm}$ of edge, hence, a part of dominant nodule was considered untreated; 10 patients had dominant nodule completely treated, but had additional nodules
}

Figure 2 Simulation, pictorial representation and outcomes of focal HIFU in the whole-mount radical prostatectomy specimens of patients eligible for focal therapy; $n=25$ patients (IDEAL stage 0 study). HIFU, high-intensity focused ultrasound.

left behind after simulated MPP, while $86 \%$ would have avoided whole-gland therapy.

IDEAL stage 1/2a: baseline characteristics, modifications to technique and outcomes

Baseline characteristics are provided in table 1. Eight patients underwent MPP. Median (IQR) age and PSA were $54(52-57)$ years and $4.4(3.8-6.1) \mathrm{ng} / \mathrm{mL}$, respectively. All patients were potent preoperatively with a median IIEF-5 score of 24 . The majority of the patients had cT1 disease, $62.5 \%(\mathrm{n}=5)$. The biopsy Gleason score was $3+4$ in $50 \%(\mathrm{n}=4)$; the remaining 4 had $3+3$ disease. The median (IQR) number of cores positive on biopsy were $3.5(2-5)$. Seventy-five $(n=6)$ per cent of the patients had unilateral disease on biopsy. None of the eight patients met the Epstein criteria for active surveillance. ${ }^{25}$

Two surgeons (MM and WJ) were involved in the procedure, with MM being the supervising surgeon in all cases. Median (IQR) console time was 134 (108-148) min. Hemostasis was accomplished with a cautery: no hemostatic agents were used. There were no complications intraoperatively or postoperatively. None of the procedures were converted to conventional radical prostatectomy (table 2). One modification to technique was made during the series: intraoperative ultrasound was introduced after the first two cases, to measure the depth of residual tissue left in situ. This was between 4 and $10 \mathrm{~mm}$ in all six men, confirming that the intent had been 

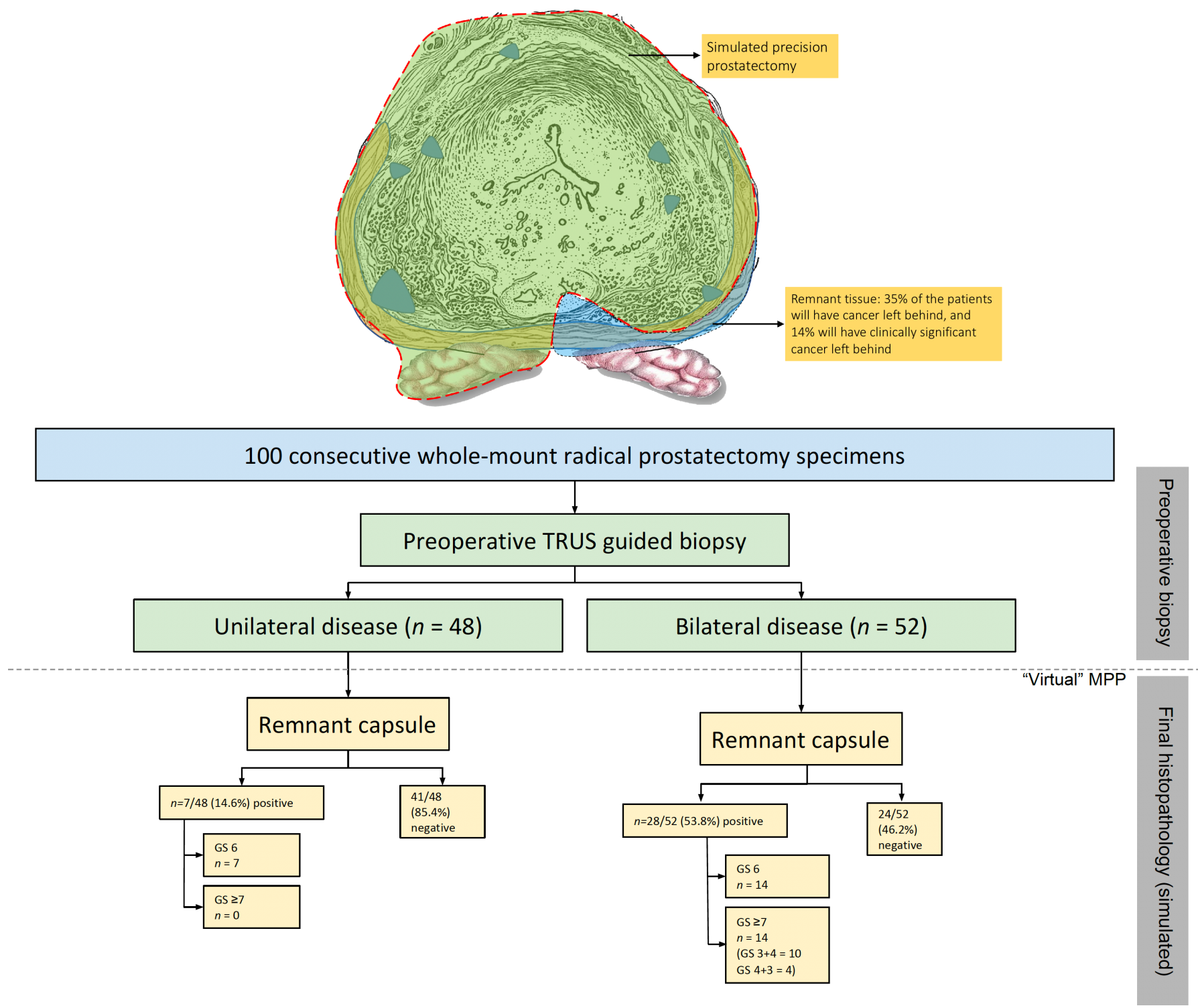

Figure 3 Simulation, pictorial representation and outcomes of precision prostatectomy in the whole-mount radical prostatectomy specimens of patients eligible for focal therapy; $n=100$ patients (IDEAL stage 0 study). GS, Gleason score; TRUS, transrectal ultrasound.

achieved. On final pathology, five men (62.5\%) had pT2c disease. No patient had a positive surgical margin on the side of the radical prostatectomy, while two patients had a positive margin at the site of precision prostatectomy, but a negative frozen section of the capsular tissue. One of the two patients with positive surgical margins on the specimen site had Gleason 3+4 disease at the affected margin, while the other had Gleason 3+3. Follow-up protocol biopsy of the remnant tissue showed focal Gleason six cancer in the former patient, and no cancer in the latter.

At 12 months from surgery, all patients had recovered urinary continence and sexual potency. The median (IQR) time to urinary continence was 1 (1-2.5) month and for sexual potency was 4 (4-10) months, with seven of the eight $(87.5 \%)$ patients potent by 4 months. The median (IQR) 12-month IPSS and IIEF-5 scores were 3 (2-4) and 21 (19-25), respectively. The median (IQR) PSA was $0.2(0.1-0.4) \mathrm{ng} / \mathrm{mL}$ at 12 months, and the PSAs remained stable at 24-30 months of follow-up. Per the AUA definition for biochemical recurrence following radical prostatectomy, two of the eight (25\%) patients had biochemical recurrence within 12 months (table 2). None $(0 \%)$ of the patients would have been considered to have a biochemical recurrence, per the ASTRO criteria. ${ }^{26}$ The PSA values of the two patients with BCR (per the AUA criteria) were 0.4 and $0.7 \mathrm{ng} / \mathrm{mL}$ at 12 months.

Two men with an undetectable PSA and excellent functional outcomes declined protocol biopsies. Four men had protocol biopsies in the absence of PSA recurrence, and none had evidence of cancer (median (range) cores: 8 (8-15)). Two patients with PSA recurrence (one with a positive and one with a negative surgical margin) underwent prostate transrectal ultrasound-guided biopsy at 12 months. Both patients had focal Gleason 3+3 cancer in the remnant and are on active surveillance. All four patients without biochemical recurrence had a 
Table 1 Preoperative characteristics in patients undergoing robot-assisted precision prostatectomy, $n=8$ (MPP; IDEAL stage $1 / 2 a)$

\section{PREOPERATIVE PARAMETERS}

$\begin{array}{ll}\text { Demographic details } & \\ \text { Age in years; median (IQR) } & 54(52-57) \\ \text { BMI in kg/m²; median (IQR) } & 29(24.7-34.3) \\ \text { Comorbidities; n (\%) } & 2(25) \\ \text { Diabetes mellitus } & 1(12.5) \\ \text { Hypertension } & 0(0) \\ \text { COPD/Asthma } & 2(25) \\ \text { Cerebrovascular disease } & 0(0) \\ \text { Prior abdominal surgeries } & 2(1-3) \\ \text { American Society of Anesthesiologists } & \\ \text { score; median (IQR) } & \\ \text { Tumor characteristics } & 4.4(3.8-6.1) \\ \text { PSA in ng/mL; median (IQR) } & \\ \text { Clinical tumor stage; } n \text { (\%) } & 5(62.5) \\ \text { cT1 } & 3(37.5) \\ \text { cT2a }\end{array}$

Transrectal ultrasound-guided biopsy

(12-16 cores)

$\begin{array}{ll}\text { Highest Gleason score; } n(\%) & 5(62.5) \\ 3+3 & 4(50) \\ 3+4 & 4(50) \\ \begin{array}{l}\text { Number of positive cores; median } \\ \text { (IQR) }\end{array} & 3.5(2-5) \\ \text { Percent core positivity; median (IQR) } & 29.2(16.7-41.7) \\ \begin{array}{l}\text { Unilateral disease (core positivity } \\ \text { limited to one side); } n \text { (\%) }\end{array} & 6(75) \\ \text { Preoperative IIEF-5 score; median (IQR) } & 24(23-25) \\ \text { Preoperative IPSS score; median (IQR) } & 3(2.5-3.5)\end{array}$

$\mathrm{BMI}$, bodymass index; COPD, chronic obstructive pulmonary disease; IIEF-5, International Index of Erectile Function; IPSS, International Prostate Symptom Score; IQR, IQR range; PSA, prostate specific antigen.

combination of benign prostatic tissue and fibromuscular tissue on biopsies. No patient had required additional therapy at their last follow-up (24-30 months).

\section{DISCUSSION}

Radical prostatectomy or radiation therapy are well-established methods of treating CaP. A high rate of cancer control is achieved, but at the cost of significant functional effects, especially erectile dysfunction. Two contemporary publications suggest that erectile dysfunction remains problematic. Published data from well-designed prospective trials in the UK (ProtecT) ${ }^{27}$ and the USA (CAESAR) ${ }^{1}$ demonstrate erectile dysfunction rates of $50 \%-70 \%$ at 12 months after prostatectomy. A report from a single center
Table 2 Operative and postoperative outcomes in patients undergoing robot-assisted precision prostatectomy, $\mathrm{n}=8$ (MPP; IDEAL stage 1/2a)

\section{OPERATIVE OUTCOMES}

Operative time (incision-closure) in minutes; median 173 (143-185) (IQR)

Console time (console start-finish) in minutes; median (IQR)

$134(108-148)$

Patients with suprapubic tube at end of procedure; 7 (87.5) $\mathrm{n}(\%)$

Estimated blood loss; median (IQR) $100(85-200)$

Volume of prostate tissue left behind in ${ }^{*} \mathrm{~cm} \wedge 3$; $\quad 4(2-7)$ median (IQR)

Complications/adverse outcomes; $\mathrm{n}(\%)$

Intraoperative complications

$0(0)$

Margin positivity of the remnant tissue on frozen $\quad 0(0)$ section analysis

Need for conversion to radical prostatectomy

$0(0)$

\section{POSTOPERATIVE OUTCOMES}

$\begin{array}{ll}\text { Length of stay in days; median (IQR) } & 1(1-1) \\ \text { Postoperative complications }{ }^{\star \star} ; n(\%) & 0(0) \\ \text { Hospital readmission in } 30 \text { days; } n(\%) & 0(0) \\ \text { Pathological analysis; } \mathrm{n}(\%) & \\ \text { Pathological tumor stage; } \mathrm{n}(\%) & 3(37.5) \\ \text { pT2a } & 5(62.5) \\ \text { pT2c } & 1(12.5) \\ \text { Highest Gleason score; } \mathrm{n}(\%) & 6(75) \\ \text { 3+3 } & 1(12.5) \\ \text { 3+4 } & 0(0) \\ 4+3 & \end{array}$

Lymph-node involvement; $\mathrm{n}(\%)$

Nerve sparing on the side of the dominant nodule; $\mathrm{n}(\%)$

\begin{tabular}{|c|c|}
\hline Veil & $5(62.5)$ \\
\hline Standard & $3(37.5)$ \\
\hline \multicolumn{2}{|l|}{ Surgical margin positivity; $\mathrm{n}(\%)$} \\
\hline On the side of the radical dissection & $0(0)$ \\
\hline On the side of the precision prostatectomy & $2(25)$ \\
\hline PSA at 12 months; median (IQR) & $0.2(0.1-0.4)$ \\
\hline $\begin{array}{l}\text { Patients with biochemical recurrence per AUA } \\
\text { definition; } n(\%)\end{array}$ & $2(25)$ \\
\hline $\begin{array}{l}\text { Patients with biochemical recurrence per ASTRO } \\
\text { criteria; } \mathrm{n}(\%)\end{array}$ & $0(0)$ \\
\hline $\begin{array}{l}\text { Patients undergone additional therapy at latest } \\
\text { follow-up ( } 24-30 \text { months); } n(\%)\end{array}$ & $0(0)$ \\
\hline Urinary continent at 12 months; $n$ (\%) & $8(100)$ \\
\hline \multicolumn{2}{|l|}{ Sexual health at 12 months; $n(\%)$} \\
\hline Potent at 12 months ${ }^{\star \star \star}$ & $8(100)$ \\
\hline Using PDE-5 inhibitors & $4(50)$ \\
\hline Using intracavernosal injections & $0(0)$ \\
\hline
\end{tabular}

${ }^{*} \mathrm{n}=6$ for this data point; **Postoperative complications included need for blood transfusion, DVT/PE, lymphoceles, urinary tract infection or cardiopulmonary events; *** Five out of the eight patients were potent by 1 month (total $=62.5 \%$ ), an additional two were potent by 4 month (total $=87.5 \%)$, and all were potent by 12 months $(100 \%)$.

AUA, American Urological Association; DVT, deep vein thrombosis; PE, pulmonary embolism; PSA, prostate-specific antigen. 
of excellence in the USA, Memorial Sloan-Kettering Cancer Center, is largely confirmatory (27\% potency), despite the use of intracavernosal injections, refinements in nerve sparing, and adoption of robotics. ${ }^{2}$

The results are quite different in men undergoing simple prostatectomy or prostate-sparing radical cystectomy. Several series report a potency rate of about $90 \%$. This suggests that a key to preserving potency may lie in preserving the "prostatic capsule", ${ }^{28-30}$ where the network of erectogenic nerves is located. ${ }^{21}{ }^{22}$ In this regard, focal HIFU, which spares at least $50 \%$ of the prostate, ${ }^{10-12}$ has shown promise. The seminal work by Ahmed and colleagues reported a potency rate of $89 \%$ at 12 months and a continence rate of $100 \% .{ }^{10}$ Other studies on focal therapy (HIFU and others) have corroborated their findings. 111331

There remains a concern regarding the oncological efficacy of the procedure, however. A multicenter 5-year follow-up of 505 men with intermediate risk $\mathrm{CaP}$ who underwent focal HIFU reported a cancer-specific survival of $100 \%,{ }^{12}$ but a retreatment rate of $27 \%$ and a positive biopsy rate of $25 \%$ in men who were re-biopsied. Another single-institution study of 150 men ( $\mathrm{n}=132$, Gleason 7) showed residual cancer in $81 \%$ of men who underwent confirmatory biopsy and a $25 \%$ retreatment rate, despite an attempt to ablate fivefold to 10 -fold the lesion volume detected on mp-MRI. ${ }^{13}$

We reasoned that the high anatomical failure rate from focal HIFU may be related to the presence of residual $\mathrm{CaP}$ that is below the detection resolution of MRI, currently, $10 \mathrm{~mm}$ in diameter or $0.5 \mathrm{~cm} \wedge 3$ in volume. Our preclinical, stage 0 , prostatic mapping study was performed to test the validity of this assumption. We found that $>70 \%$ of men who were candidates for focal or hemiablation therapy harbored low volume clinically significant CaP on analysis of whole-mount specimens. These results are comparable with other reports. Kenigsberg et al in a simulation study of focal HIFU in radical prostatectomy specimens noted that significant cancer (Gleason pattern 4 or above) would have been left behind in $23.7 \%$ of the patients who had underwent mp-MRI evaluation. ${ }^{32}$ Similarly, Elkhoury et al demonstrated that detection rate of Gleason $\geq 7$ cancer via mp-MRI fusion biopsy varies between $47 \%$ and $60 \%$ in patients with mp-MRI visible lesions, while $15 \%$ of clinically significant cancers are invisible. $^{33}$

Our analysis allowed us to simulate a novel surgical technique, precision prostatectomy. In this approach, we attempt to remove all prostatic tissue, but for a $5-10 \mathrm{~mm}$ rim of prostate capsule on the side contralateral to the dominant lesion. In a "what-if" analysis, if all men had undergone precision prostatectomy, the residual cancer rate would have been $14 \%$ (10\% Gleason 3+4, 4\% Gleason $4+3$ ) while it would have been $68 \%$ had they undergone focal HIFU (44\% Gleason 3+4, $16 \%$ Gleason 4+3, $4 \%$ Gleason $4+4 \%$ and $4 \%$ Gleason $4+5$; see figures 2 and $3)$. Looked at in another way, precision prostatectomy would have spared $86 \%$ of men from having whole-gland therapy, with equivalent oncologic control, and a higher erectile function rate than whole-gland therapy. This is probably due to the fact that precision prostatectomy allows for removal of greater than $90 \%$ of the prostate with complete removal of the side of the dominant lesion, and removal of the majority of satellite lesions on the contralateral side.

Following IDEAL guidelines, the stage 0 study was followed by a stage 1/2a study of MPP in eight highly selected patients. Precision prostatectomy offers several conceptual benefits over focal/hemiablation. No patient needed to be excluded because of prostate size or the location of the tumor. It is noteworthy that manufacturers' guidelines suggest limiting focal HIFU to men with prostate volumes less than $40 \mathrm{cc}$, and to men without apical/anterior tumors, approaching $75 \%$ of men in the preclinical study. Further, the treated tissue remains available for detailed pathological or genomic analysis after MPP, while it is destroyed at ablation.

In this development study, we demonstrate that MPP is technically feasible and safe. No patient needed to be converted to radical prostatectomy, or suffered an intraoperative or postoperative complication. Only one modification to technique was made during this series: using intraoperative ultrasonography with a drop-in probe, we were able to measure accurately the volume of residual tissue and confirm that the intent of removing $>90 \%$ of prostatic tissue was met.

From a functional outcomes standpoint, the patients undergoing precision prostatectomy achieved excellent results, with all eight $(100 \%)$ patients achieving urinary continence within 4 months of surgery and potency within 12 months.

Evaluation of the oncological effectiveness of focal therapy has been bedeviled by a lack of consensus on what constitutes a PSA failure, an important surrogate for oncological control after radical prostatectomy. After all, a significant portion of the prostate is left behind after focal therapy, and continues to produce PSA. Most investigators are silent about PSA values after focal therapy, but Bass et al found that the PSA nadir was $2-3 \mathrm{ng} / \mathrm{mL}$ at 2-3 years of follow-up. ${ }^{13}$ Using the AUA definition for biochemical recurrence following radical prostatectomy (PSA of $0.2 \mathrm{ng} / \mathrm{mL}$ and rising, or a single value of $0.4 \mathrm{ng} /$ $\mathrm{mL}^{24}$ ), we demonstrated that two out of the eight $(25 \%)$ patients developed biochemical recurrence, whereas no patient $(0 \%)$ had a recurrence if we used the ASTRO criteria for recurrence. ${ }^{26}$ Postoperative biopsy in the two patients with biochemical recurrence (per AUA criteria) showed low-volume, low-risk disease: $1 / 6$ cores of Gleason $3+3$ in one patient and 2/6 cores of Gleason $3+3$ in the other.

Another important oncological consideration is the evaluation of surgical margins. Information about surgical margins cannot be obtained from ablation studies, where, by definition, no tissue is removed. Further, the use of surgical margins as a meaningful endpoint is debatable: the recent 29-year follow-up of SPCG-4 trial, ${ }^{34}$ and our 
prior work, ${ }^{35}$ suggests that surgical margins matter minimally, if at all, unless patients have extraprostatic disease and high-grade cancer. In line with this, the two patients that had positive surgical margins have not required additional treatment and remain on active surveillance with stable PSAs.

Our study has several limitations. By design, this is a stage $0-1 / 2 \mathrm{a}$, single-center study evaluating a small number of patients. Hence, conclusions should be drawn with caution. However, the study design is in accordance with recommendations provided by the IDEAL collaboration for surgical innovation. A second limitation is that MRIs were not performed in these men. The reasons for this were partly pragmatic, and partly by intent. At the time that the patients in the stage 0 study were operated on, mp-MRI was not readily available for general clinical use in the USA. And a raison d'etre for the study was to examine the distribution of clinically significant cancers that are smaller than the current detection limit of mp-MRI in a target population that would qualify for focal therapy.

Another limitation of precision prostatectomy is that two out of eight patients had residual cancer on protocol biopsy. Although these patients have not required any further treatment as of the writing of this paper, it suggests that there is room for improvement in selection of the patients. For the reasons explained above, we are not convinced that mp-MRI would eliminate the rate of residual cancer, as this study and others suggest that a substantial proportion of clinically significant tumors are below the detection limits of mp-MRI. ${ }^{18} 323336$ We are exploring an alternate diagnostic methodology to accurately stage focal therapy candidates preoperatively with 3D transperineal saturation biopsy. ${ }^{37}$ Biopsies that target specifically the area of the prostate that will be left behind during precision prostatectomy may complement biopsies that target the tissue that is removed, and thus reduce the incidence of missed cancer.

It can also be argued that cancer control requires the treatment of just the index lesion. This conclusion is controversial. Kneppers et al showed that in about $20 \%$ of men, lymph node metastases arose from non-index lesions. ${ }^{38}$ In a more recent study, the Palapattu group demonstrated that, using next generation sequencing, the genetic footprint of MRI invisible lesions was indistinguishable from MRI visible or index lesions. ${ }^{39}$ Further, the short duration of follow-up and the high incidence of secondary treatment after focal HIFU argue for the development of newer approaches, a better mouse trap, if you will. It seems reasonable to plead that if new procedures are being contemplated, their development should follow a structured pattern, the IDEAL path.

Finally, conclusions about functional superiority are best resolved with randomized clinical trials. We are in the process of doing such a trial. The data reported here merely form a basis of such a trial.

\section{CONCLUSIONS}

Whole-gland therapy of CaP provides excellent cancer control, but results in a high rate of erectile dysfunction. Focal ablative therapy eliminates sexual dysfunction, but at the cost of leaving residual cancer. We propose a novel surgical approach to localized CaP similar to the surgical treatment of breast cancer. Precision prostatectomy is feasible, safe, offers excellent postoperative functional recovery, and promises superior oncological control (compared to ablative technologies). For now, precision prostatectomy may defer or avoid the need for immediate whole-gland therapy in $80 \%$ of men with intermediate-risk CaP, while reducing the risk of postoperative erectile dysfunction. However, longer follow-up with randomized trials is necessary to properly define the exact role of precision prostatectomy in the treatment of men with localized CaP.

Contributors Study design and conception: MM. Administrative support: MM. Data collection, analysis and interpretation: AS, WJ, KT, FA, IPZ, SA, NG, MM. Drafting of the manuscript: AS, MM. Revision of the manuscript: AS, MM.

Funding The authors have not declared a specific grant for this research from any funding agency in the public, commercial or not-for-profit sectors.

Competing interests None declared.

Patient consent for publication Not required.

Provenance and peer review Commissioned; externally peer reviewed.

Data availability statement Data are available on request.

Open access This is an open access article distributed in accordance with the Creative Commons Attribution Non Commercial (CC BY-NC 4.0) license, which permits others to distribute, remix, adapt, build upon this work non-commercially, and license their derivative works on different terms, provided the original work is properly cited, appropriate credit is given, any changes made indicated, and the use is non-commercial. See: http://creativecommons.org/licenses/by-nc/4.0/.

\section{REFERENCES}

1. Barocas DA, Alvarez J, Resnick MJ, et al. Association between radiation therapy, surgery, or observation for localized prostate cancer and patient-reported outcomes after 3 years. JAMA 2017;317:1126-40.

2. Capogrosso P, Vertosick EA, Benfante NE, et al. Are we improving erectile function recovery after radical prostatectomy? analysis of patients treated over the last decade. Eur Urol 2019;75:221-8.

3. Fisher B, Anderson S, Bryant J, et al. Twenty-Year follow-up of a randomized trial comparing total mastectomy, lumpectomy, and lumpectomy plus irradiation for the treatment of invasive breast cancer. N Engl J Med 2002;347:1233-41.

4. Vaidya JS, Joseph DJ, Tobias JS, et al. Targeted intraoperative radiotherapy versus whole breast radiotherapy for breast cancer (TARGIT-A trial): an international, prospective, randomised, noninferiority phase 3 trial. The Lancet 2010;376:91-102.

5. Van Poppel H, Da Pozzo L, Albrecht W, et al. A prospective, randomised EORTC intergroup phase 3 study comparing the oncologic outcome of elective nephron-sparing surgery and radical nephrectomy for low-stage renal cell carcinoma. Eur Urol 2011;59:543-52.

6. Bissada NK, Yakout HH, Fahmy WE, et al. Multi-Institutional long-term experience with conservative surgery for invasive penile carcinoma. J Urol 2003;169:500-2.

7. van der Poel HG, van den Bergh RCN, Briers E, et al. Focal Therapy in Primary Localised Prostate Cancer: The European Association of Urology Position in 2018. Eur Urol 2018;74:84-91.

8. Liu W, Laitinen S, Khan S, et al. Copy number analysis indicates monoclonal origin of lethal metastatic prostate cancer. Nat Med 2009;15:559-65.

9. Guo CC, Wang Y, Xiao L, et al. The relationship of TMPRSS2-ERG gene fusion between primary and metastatic prostate cancers. Hum Pathol 2012;43:644-9. 
10. Ahmed HU, Hindley RG, Dickinson L, et al. Focal therapy for localised unifocal and multifocal prostate cancer: a prospective development study. Lancet Oncol 2012;13:622-32.

11. Valerio M, Ahmed HU, Emberton $\mathrm{M}$, et al. The role of focal therapy in the management of localised prostate cancer: a systematic review. Eur Urol 2014;66:732-51.

12. Guillaumier S, Peters M, Arya M, et al. A multicentre study of 5-year outcomes following focal therapy in treating clinically significant nonmetastatic prostate cancer. Eur Urol 2018;74:422-9.

13. Bass R, Fleshner N, Finelli A, et al. Oncologic and functional outcomes of partial gland ablation with high intensity focused ultrasound for localized prostate cancer. J Urol 2019;201:113-9.

14. Barkun JS, Aronson JK, Feldman LS, et al. Evaluation and stages of surgical innovations. The Lancet 2009;374:1089-96.

15. Ergina PL, Cook JA, Blazeby JM, et al. Challenges in evaluating surgical innovation. The Lancet 2009;374:1097-104.

16. McCulloch P, Altman DG, Campbell WB, et al. No surgical innovation without evaluation: the ideal recommendations. The Lancet 2009;374:1105-12

17. Golan R, Bernstein AN, McClure TD, et al. Partial gland treatment of prostate cancer using high-intensity focused ultrasound in the primary and salvage settings: a systematic review. J Urol 2017:198:1000-9.

18. Bratan F, Melodelima C, Souchon R, et al. How accurate is multiparametric MR imaging in evaluation of prostate cancer volume? Radiology 2015;275:144-54.

19. Menon M, Shrivastava A, Kaul S, et al. Vattikuti Institute prostatectomy: contemporary technique and analysis of results. Eur Urol 2007:51:648-58. discussion 57-8.10.1016/..eururo.2006.10.055

20. Ghani KR, Trinh Q-D, Menon M. Vattikuti Institute ProstatectomyTechnique in 2012. J Endourol 2012;26:1558-65.

21. Clarebrough EE, Challacombe BJ, Briggs $\mathrm{C}$, et al. Cadaveric analysis of periprostatic nerve distribution: an anatomical basis for high anterior release during radical prostatectomy? Journal of Urology 2011;185:1519-25

22. Costello AJ, Brooks M, Cole OJ. Anatomical studies of the neurovascular bundle and cavernosal nerves. BJU Int 2004;94:1071-6.

23. Ruijter E, van Leenders G, Miller G, et al. Errors in histological grading by prostatic needle biopsy specimens: frequency and predisposing factors. J Pathol 2000;192:229-33.

24. Thompson IM, Valicenti RK, Albertsen P, et al. Adjuvant and salvage radiotherapy after prostatectomy: AUA/ASTRO guideline. $J$ Urol 2013;190:441-9.

25. Dall'Era MA, Albertsen PC, Bangma C, et al. Active surveillance for prostate cancer: a systematic review of the literature. Eur Urol 2012;62:976-83
26. Roach M, Hanks G, Thames $\mathrm{H}$, et al. Defining biochemical failure following radiotherapy with or without hormonal therapy in men with clinically localized prostate cancer: recommendations of the RTOGASTRO Phoenix consensus conference. Int J Radiat Oncol Biol Phys 2006:65:965-74.

27. Donovan JL, Hamdy FC, Lane JA, et al. Patient-Reported outcomes after monitoring, surgery, or radiotherapy for prostate cancer. N Engl $J$ Med Overseas Ed 2016;375:1425-37.

28. Hanbury DC, Sethia KK. Erectile function following transurethral prostatectomy. Br J Urol 1995;75:12-13.

29. Davila HH, Weber T, Burday D, et al. Total or partial prostate sparing cystectomy for invasive bladder cancer: long-term implications on erectile function. BJU Int 2007;100:1026-9.

30. Sood A, Abdollah F, Menon M. Je Le pansai, Dieu Le guerit. Eur Urol 2017;72:343-4.

31. Bahn D, de Castro Abreu AL, Gill IS, et al. Focal cryotherapy for clinically unilateral, low-intermediate risk prostate cancer in 73 men with a median follow-up of 3.7 years. Eur Urol 2012;62:55-63.

32. Kenigsberg AP, Llukani E, Deng F-M, et al. The use of magnetic resonance imaging to predict oncological control among candidates for focal ablation of prostate cancer. Urology 2018;112:121-5.

33. Elkhoury FF, Felker ER, Kwan L, et al. Comparison of targeted vs systematic prostate biopsy in men who are biopsy naive: the prospective assessment of image registration in the diagnosis of prostate cancer (PAIREDCAP) study. JAMA Surg 2019. doi:10.1001/ jamasurg.2019.1734. [Epub ahead of print: 12 Jun 2019].

34. Bill-Axelson A, Holmberg L, Garmo H, et al. Radical prostatectomy or watchful waiting in prostate cancer -29 -Year follow-up. N Engl J Med 2018;379:2319-29.

35. Abdollah F, Moschini M, Sood A, et al. When should a positive surgical margin ring a Bell? an analysis of a multi-institutional robotassisted laparoscopic radical prostatectomy database. J Endourol 2016;30:201-7

36. Le JD, Tan N, Shkolyar E, et al. Multifocality and prostate cancer detection by multiparametric magnetic resonance imaging: correlation with whole-mount histopathology. Eur Urol 2015;67:569-76.

37. Crawford ED, Rove KO, Barqawi AB, et al. Clinical-Pathologic correlation between transperineal mapping biopsies of the prostate and three-dimensional reconstruction of prostatectomy specimens. Prostate 2013;73:778-87.

38. Kneppers J, Krijgsman O, Melis M, et al. Frequent clonal relations between metastases and non-index prostate cancer lesions. $\mathrm{JCl}$ Insight 2019;4.

39. Simpa Salami S S, Nallandhighal S, Takhar M, et al. Biologic significance of magnetic resonance imaging Invisibility in localized prostate cancer. JCO Precision Oncology 2019;3. 